\title{
Residua Back Pain after Vertebroplasty
}

\section{TO THE EDITOR:}

Currently, cement leakage is deemed to be the main complication of percutaneous vertebroplasty (PVP), which may lead to compression of neural structures, fracture in adjacent levels, and disc degeneration or embolism; and intravertebral pressure (IP) has been described as an important factor determining the risk for cement leakage by different authors. However, residual back pain after PVP is sometimes encountered by pain physicians. We will report on a patient with residual back pain after PVP, whose back pain was progressive in first 3 days and was eliminated after one month using sustained immobilization and pain medication. The increased IP is discussed as a possible reason. As the volume of each patient's compressive vertebral body is different, filling the compressed vertebra with adequate cement and providing enough stiffness and strength warrants further study. A 60-year-old woman presented to the hospital with thoracic back pain for approximately 2 weeks following an accidental tumble. The pain was reported to be a dull pain that was a constant 7 of 10 in severity and was alleviated slightly when lying supine. There was no accompanying radiating pain or paresthesia. Recent roentgenographic examination and magnetic resonance imaging confirmed the diagnosis of acute osteoporotic compression fracture in T10 (Fig. 1).
Percutaneous vertebroplasty (PVP) was performed at the T10 level under local anesthesia. The procedure was accessed using a 13-gauge trocar placed via a left transpedicular approach. Polymethylmethacrylate (PMMA) was injected into the vertebral body through the trocar. A total of $7 \mathrm{~mL}$ of PMMA was used to achieve a satisfactory vertebral body filling without a visible cement extravasation, which was confirmed by the anteroposterior and lateral fluoroscopy (Fig. 2). Of note, when approximately $6 \mathrm{~mL}$ of PMMA had been injected, it was observed that the thoracic back pain was aggravated. Postoperatively, this pain was progressive in first 3 days, which once reached 9 using a visual analogue scale. Under the sustaining immobilization and pain medication, it was eliminated after one month.

Currently, cement leakage is deemed to be the main complication of PVP, which may lead to compression of neural structures, fracture in adjacent levels, and disc degeneration or embolism; and intravertebral pressure (IP) has been described as an important factor in determining the risk for cement leakage by different authors (1-6). However, residual back pain after PVP is sometimes encountered by pain physicians. Based on the clinical study in which Wang et al (6) found that the IP of a compressed vertebra with an intact shell was

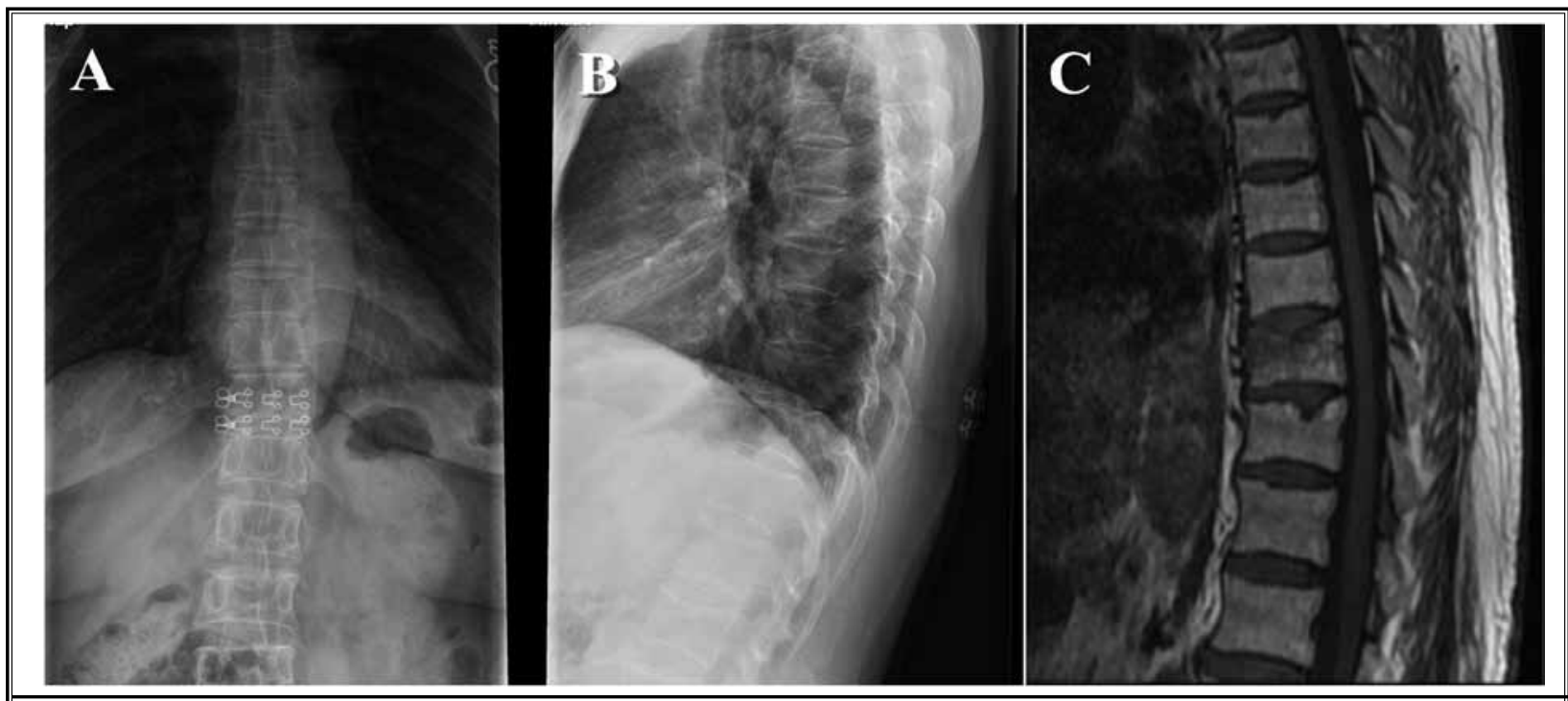

Fig. 1. Panel $A$ and $B$ showing the preoperative $x$-ray of the thoracolumbar spine including the anteroposterior (Panel $A)$ and lateral (Panel B) views. Panel C showing a preoperative axial T1-weighted magnetic resonance image. 


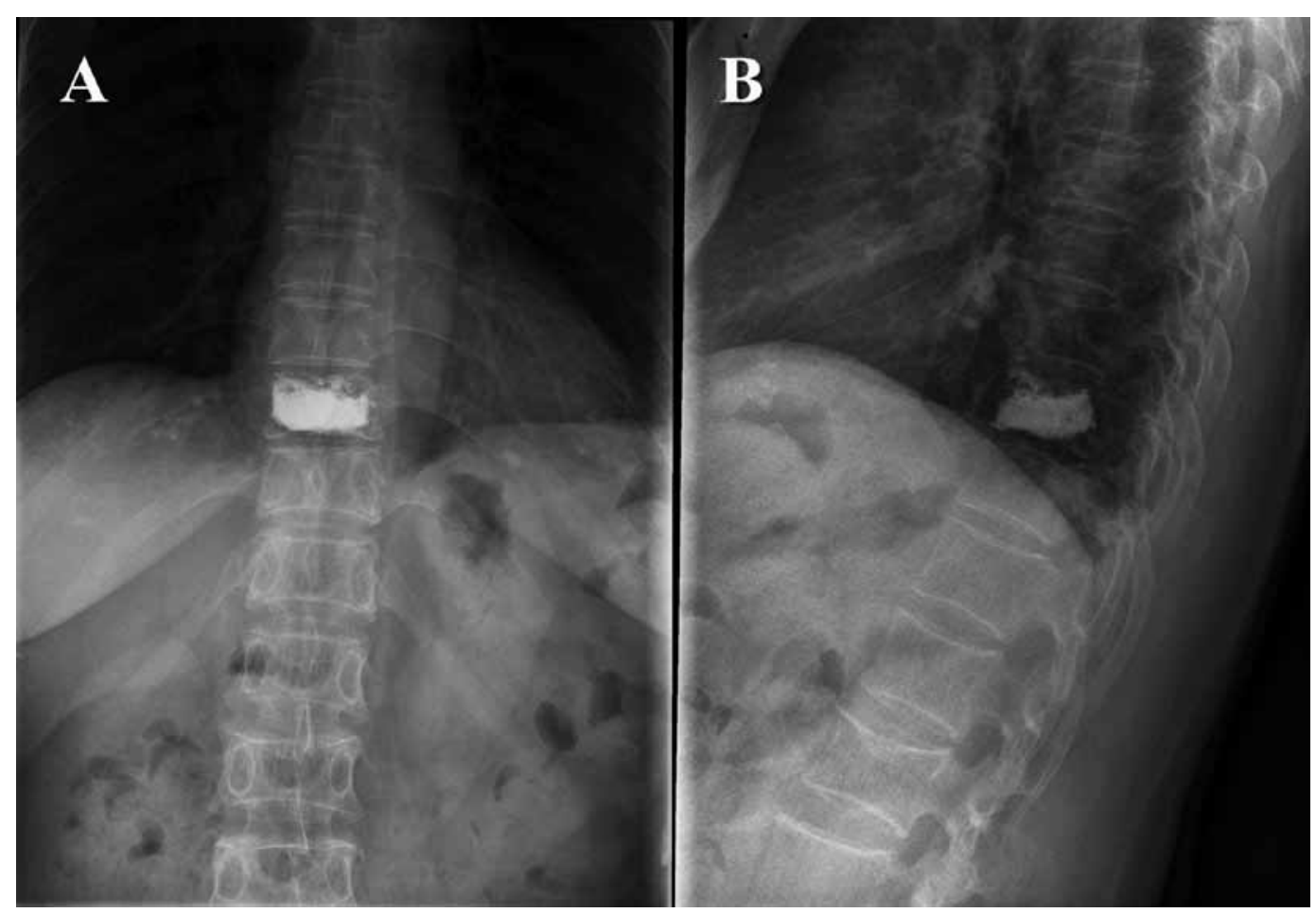

Fig. 2. Panel $A$ and $B$ showing the postoperative $x$-ray of the thoracolumbar spine including the anteroposterior (Panel $A)$ and lateral (Panel B) views.

significantly higher than that of an adjacent normal vertebrae, we considered that the increased IP could contribute to causing the back pain after vertebral compressive fracture and this residual back pain could also be associated with the increased IP.

As we know, PVP is regarded as a procedure providing almost immediate pain relief when treating osteoporotic or osteolytic fractures (7). The possible mechanisms causing PMMA to produce an analgesic effect involve an action to mechanically stabilize the vertebral microfractures, an exothermic reaction during the polymerization of the cement, and a neurotoxic effect of the monomer to the surrounding nervous end structures $(8,9)$. In addition, we considered that the puncture in the process of PVP could create a vent in the compressive vertebra, which helps to decrease the IP and then to relieve pain. In studies measuring IP in PVP or percutaneous kyphoplasty (PKP), the dramatic increase of IP was observed in the process of injecting cement (10-12). However, only one vivo study described the IP before cement injection and after injection simultaneously (6), in which a higher IP was recorded after cement injection. Hence, we considered that the residual back pain in this case was related to the remaining high IP after PVP.

In the studies to date, the volume and viscosity of cement, injected flow rate, the integrity of vertebral shell, and bone mineral density are proposed as potential factors affecting IP (6). However, there are still different views about whether one of these factors could affect IP $(3,10,12-14)$. To explain the difference between these published reports, we believe that there is an interaction between the aforementioned factors affecting IP. Single factor analysis is not appropriate for detecting its actual influence on IP. In addition, most of the current results are derived from cadaver studies; the samples of clinical studies are relatively small. Whether these conclusions are applicable in vivo study needs to be verified in further comparative studies with a high number of patients. We think that IP is remarkably increased when injecting the cement with a relatively rapid speed into a comparably small vertebra with an intact shell, notably when the void within vertebra has been filled with cement. It is well known that the constituents of the vertebral body primarily consist of intramedullary fat and blood. When the vertebra is compressed, an obvious medullary edema exists in the vertebra accompanied by congestion. The injected cement first fills the blood sinus 
within vertebra, and then squeezes the intramedullary fat causing a significant increase in IP. Although the cement injected into the compressed vertebra is beneficial for restoring vertebra stiffness and strength, it is notable that the excessive cement could not only increase the risk for cement leakage but also lead to residual pain. In this case, when the excessive cement was injected, it generated a remarkable increase in IP which was reduced gradually with the elimination of the medullary edema. This explains why the patient suffered a month-long back pain after PVP, which was even aggregated in first 3 days postoperatively.

Balancing the relationship between injecting an ideal volume of cement and acquiring satisfactory stiffness and strength of the vertebral body is significant when performing PVP and helps prevent postoperative residual back pain. As there is an difference in the volume of each patient's compressed vertebral body, filling the compressive vertebra with adequate cement and providing enough stiffness and strength warrants further study.

\section{Jun-Song Yang, MD \\ Department of Spinal Surgery \\ Hong-Hui Hospital \\ Medical College of Xi'an Jiaotong University}

\section{References}

1. Yeom JS1, Kim WJ, Choy WS, Lee CK, Chang BS, Kang JW. Leakage of cement in percutaneous transpedicular vertebroplasty for painful osteoporotic compression fractures. J Bone Joint Surg $\mathrm{Br}$ 2003; 85:83-89.

2. Hulme PA1, Krebs J, Ferguson SJ, Berlemann U. Vertebroplasty and kyphoplasty: A systematic review of 69 clinical studies. Spine (Phila Pa 1976) 2006; 31:1983-2001.

3. Ryu KS, Park CK, Kim MC, Kang JK. Dose-dependent epidural leakage of polymethylmethacrylate after percutaneous vertebroplasty in patients with osteoporotic vertebral compression fractures. J Neurosurg 2002; 96:56-61.

4. Sonmez E, Yilmaz C, Caner H. Development of lumbar disc herniation following percutaneous vertebroplasty. Spine (Phila Pa 1976) 2010; 35:E93-E95.

5. Nagaraja S, Awada HK, Dreher ML, Gupta S, Miller SW. Vertebroplasty increases compression of adjacent IVDs and vertebrae in osteoporotic spines. Spine J 2013; 13:1872-1880.

6. Wang Y, Huang F, Chen L, Ke ZY, Deng ZL. Clinical measurement of ntravertebral pressure during vertebroplasty and kyphoplasty. Pain Physician 2013; 16:E411-E418.

7. Chandra RV, Yoo AJ, Hirsch JA. Vertebral augmentation: Update on safety, efficacy, cost effectiveness and increased survival? Pain Physician 2013; 16:309-320.

8. Biscevic M, Hamzaoglu A, Ljuca F, Gavrankapetanovi I, Nadarevi A, Rejec-Smrke B, Smrke D.Minimally invasive

\author{
Xi'an, 710054, China \\ Ding-Jun Hao, MD, PhD \\ Department of Spinal Surgery \\ Hong-Hui Hospital \\ Medical College of Xi'an Jiaotong University \\ Xi'an, 710054, China \\ Email: dingjun.hao@qq.com \\ Yuan-Ting Zhao, MD \\ Department of Spinal Surgery \\ Hong-Hui Hospital \\ Medical College of Xi'an Jiaotong University \\ Xi'an, 710054, China \\ Tuan-Jiang Liu, MD \\ Department of Spinal Surgery \\ Hong-Hui Hospital \\ Medical College of Xi'an Jiaotong University \\ Xi'an, 710054, China
}

Li-Min He, MD

Department of Spinal Surgery

Hong-Hui Hospital

Medical College of Xi'an Jiaotong University

Xi'an, 710054, China

surgery of pathologic spine fractures - vertebroplasty and kyphoplasty at department for orthopedics and traumatology of clinical centre University of Sarajevo. Medicinski Arhiv 2009; 63:234-237.

9. Yang HL, Sun ZY; Wu GZ, Chen KW, Gu Y, Qian ZL. Do vertebroplasty and kyphoplasty have an antitumoral effect? Medical Hypotheses 2011; 76:144-151.

10. Krebs J, Ferguson SJ, Bohner M, Baroud G, Steffen T, Heini PF. Clinical measurements of cement injection pressure during vertebroplasty. Spine (Phila $\mathrm{Pa}$ 1976) 2005; 30:E118-E122.

11. Weisskopf M, Ohnsorge JA, Niethard FU. Intravertebral pressure during vertebroplasty and balloon kyphoplasty: An in vitro study. Spine (Phila Pa 1976). 2008; 33:178-182.

12. Baroud G, Vant C, Giannitsios D, Bohner M, Steffen T. Effect of vertebral shell on injection pressure and intravertebral pressure in vertebroplasty. Spine (Phila $\mathrm{Pa}$ 1976) 2005; 30:68-74.

13. Bohner M, Gasser B, Baroud G, Heini P. Theoretical and experimental model to describe the injection of a polymethylmethacrylate cement into a porous structure. Biomaterials 2003; 24:2721-2727.

14. Loeffel M, Ferguson SJ, Nolte LP, Kowal JH. Vertebroplasty: Experimental characterization of polymethylmethacrylate bone cement spreading as a function of viscosity, bone porosity, and flow rate. Spine (Phila Pa 1976) 2008; 33:1352-1359. 